\title{
Analysis of Channel Effects on Direct-Sequence and Frequency-Hopped Spread-Spectrum Acoustic Communication
}

\author{
Lee Freitag, Milica Stojanovic, Sandipa Singh, and Mark Johnson
}

\begin{abstract}
Multiuser underwater acoustic communication is one of the enabling technologies for the autonomous ocean-sampling network (AOSN). Multiuser communication allows vehicles, moorings, and bottom instruments to interact without human intervention to perform adaptive sampling tasks. In addition, multiuser communication may be used to send data from many autonomous users to one buoy with RF communications capability, which will then forward the information to shore. The two major signaling techniques for multiuser acoustic communication are phase-shift keying (PSK) direct-sequence spread-spectrum (DSSS) and frequency-shift keying (FSK) frequency-hopped spread-spectrum (FHSS). Selecting between these two techniques requires not only a study of their performance under multiuser conditions, but also an analysis of the impact of the underwater acoustic channel. In the case of DSSS, limitations in temporal coherence of the channel affect the maximum spreading factor, leading to situations that may be better suited to FHSS signals. Conversely, the multipath resolving properties of DSSS minimize the effects of frequency-selective fading that degrade the performance of FSK modulation. Two direct-sequence receivers potentially suitable for the underwater channel are presented. The first utilizes standard despreading followed by decision-directed gain and phase tracking. The second uses chip-rate adaptive filtering and phase tracking prior to despreading. Results from shallow water testing in two different scenarios are presented to illustrate the techniques and their performance.
\end{abstract}

Index Terms-Acoustic communication, CDMA, direct-sequence, frequency-hop, multipath, spread-spectrum.

\section{INTRODUCTION}

C ODE-DIVISION multiple-access communication (CDMA) is an important emerging technology for underwater acoustic networks for both civilian and military purposes. CDMA permits random, overlapping access to a shared communication channel as required in an autonomous ocean-sampling network (AOSN) scenario. Drawing from the results in RF wireless communications [1], [2], two code-division spread-spectrum signalling methods are proposed for simultaneous-access communication: phase-modulated, direct-sequence spread-spectrum (DSSS) signaling, and noncoherent frequency-hopped spread-spectrum (FHSS). DSSS uses phase-shift keying and spreads the data using codes with good

Manuscript received April 13, 2000; revised July 6, 2001.

L. Freitag, M. Johnson and S. Singh are with the Woods Hole Oceanographic Institution, Applied Ocean and Physics Laboratory, Woods Hole, MA 02543 USA (e-mail: lfreitag@whoi.edu; majohnson@whoi.edu; ssingh@whoi.edu).

M. Stojanovic is with the Massachusetts Institute of Technology, Department of Aeronautics \& Astronautics, Cambridge, MA 02139 USA (e-mail: millitsa@mit.edu).

Publisher Item Identifier S 0364-9059(01)09922-8. auto- and cross-correlation properties, while FHSS methods utilize many frequency bands with hopping patterns chosen for minimum interference between users.

While both of these techniques may be suitable for use in the underwater acoustic channel, the best choice depends upon the characteristics of the particular propagation conditions, specifically time and frequency spread. Additional considerations include the desired throughput, total transducer bandwidth, and the computational resources available at the receiver.

DSSS signaling has been used to increase the SNR per data symbol and resolve multipath components for single-user, shortrange applications [3] as well as for long-range, low SNR and multiuser communication [4], [5] . The system described in [3] utilized $10 \mathrm{kHz}$ of bandwidth and short spreading codes in order to combat multipath effects in shallow water, whereas the investigations reported in [4] and [5] include discussions of multiuser access and report favorable results in the $1-2 \mathrm{kHz}$ frequency band at long ranges for a single user.

There are tremendous variations in acoustic channels depending on source-receiver geometry, bathymetry and sound-speed profile. In addition, there are many possible system configurations depending on the number of users and the desired link protocol. The large number of possible implementations requires the system design to be as flexible as possible, accommodating few to many users in a variety of channel conditions. While reconfiguration may or may not be done automatically in the field, an actual system design should allow changing basic system parameters, in particular, the spreading rate. Given the limited bandwidth available for underwater acoustic communications systems, increasing the spreading rate inevitably reduces link throughput. In the case of slow-hop frequency-shift keying (FSK) (one tone per symbol), increasing the spreading rate means increasing the symbol time (and thus the energy per symbol), which in turn, reduces the bandwidth per symbol and provides a large set of frequencies from which to select. In a fixed-power system, the SNR will increase as the rate decreases, though the gain in transmitted energy may be offset by loss due to Doppler shift and spread. This results in diminishing returns as the symbol duration increases.

In a DSSS system, the spreading rate is increased through use of more chips per data symbol, thus increasing the SNR per symbol in additive white Gaussian noise (AWGN). However, in a time-varying multipath channel, the actual gain depends upon the stability of the propagation environment. If the channel changes appreciably during one symbol period, the gain of the receiver will be reduced, ultimately resulting in a net loss 
when the length of the spreading code is increased past a certain point. This consideration motivates the design of a receiver which compensates for channel variability prior to despreading, thus decoupling channel stability from spreading rate selection as much as possible.

The primary contributions of this paper are two new receiver designs for direct-sequence spread-spectrum signaling, and the comparison of these receivers with frequency hopped FSK modulation in the ocean. One of the receivers combines the conventional matched filter with adaptive phase and gain tracking at the symbol rate. This represents the minimum-complexity DSSS processor suitable for a channel with amplitude and phase variation. The second receiver is based on a decision-directed adaptive equalizer which operates at the chip rate. While this receiver has higher complexity, it will be shown to have better performance than the symbol-rate receiver in a time-varying multipath channel.

For both FHSS and DSSS, the in-water test results focus on the changes in receiver performance with respect to the spreading rate. In AWGN, the relation is linear, whereas in the time-varying underwater environment the gain depends upon the channel, and in the case of the adaptive receiver, on the amount of variation which is removed during the equalization process.

The paper is organized as follows. In Section II, frequency-hop spread-spectrum is reviewed. In Section III, direct-sequence spread-spectrum modulation is reviewed and the two candidate receivers are presented. In Section IV, experimental data from shallow-water testing are presented.

\section{FREQUENCY-HOPPED SPREAD-SPECTRUM}

Frequency hopping is normally employed in single-user FSK systems in order to provide channel clearing time in a multipath environment. The transmitter uses a set of pre-determined frequency bins and steps through each sequentially. The total number of frequency bins is selected such that it exceeds the maximum multipath delay, so that no bin is re-used until the channel is clear.

Multiuser frequency hopping normally uses a large number of bins which are shared by a number of users [1], [2]. Many methods of selecting patterns for each user have been developed. One commonly used method which minimizes the number of times users transmit in identical bands is to use a prime number of frequency bins and assign patterns which increment through the frequency set by $k$ where $k$ is the user number [7]. In this case, any pair of users will occupy the same bin only once per pass through the hopping pattern. The irreducible error-rate due to collisions between users requires selection of an appropriate error-correction code. A large selection of bins reduces the number of coincidences, lowers the error rate and reduces the required coding gain.

In a multiuser environment with multipath, the interference between users includes not only coincidences where users step into the same band at the same time, but where multipath from one user is present when the second user utilizes that band. The performance impact is a complex function of the multipath profile, specifically the amount of power outside the symbol pe$\operatorname{riod} T$. Thus, multiuser frequency-hopping systems in multi- path require more bins than would otherwise be required. The minimum number requires knowledge of the channel-impulse response, nominal numbers may be set using the maximum expected multipath delay and the number of users.

Maximizing the number of users within a constant bandwidth means that the number of frequency bins should be large and their resultant bandwidth relatively narrow. This corresponds to the usual methodology of setting tone duration to be long with respect to the channel clearing time so that there is little signal energy beyond the symbol period. However, the delay spread in the shallow-water channel often exceeds several tens of milliseconds, and thus the first design criterion results in many narrow bands which are subject to fading and vulnerable to small Doppler shifts.

In applications where source-receiver motion is high, the tone length is selected so that the bandwidth is much larger than the maximum expected Doppler shift. The larger the bandwidth, the greater the immunity to frequency-selective fading which may be present in the channel. Unfortunately, as the number of bins are reduced the probability of error due to collisions (direct or multipath) between users increases.

While frequency-hopping FSK is very simple to implement, it has performance limitations in frequency-selective channels. In an effort to overcome these limitations, diversity and error-correction coding are often employed [11]. Convolutional codes with long constraint lengths are often combined with interleaving to provide good performance in frequency-selective channels.

\section{DiRECT-SEQUENCE SPREAD-SPECTRUM}

A $k$-user direct-sequence spread-spectrum communication system utilizes a family of $k L$-length codes with low cross-correlation [8], one of which is used by each user to modulate a binary data stream $[0,1]$ mapped to $[-1,1]$. The $L$-length bi-phase modulated code is transmitted at the chip rate $1 / T c=B$, where $B$ is the maximum bandwidth. The resulting throughput in bits per second is $B / L$.

The simplest receiver for a direct-sequence spread-spectrum signal is the conventional single-user receiver. In this receiver, the signal is multiplied by the original code and integrated over the duration of one data symbol. This operation is known as despreading. By despreading the signal, the receiver extracts the processing gain, which is equal to the number of chips per symbol, $L$. In a static or slowly-varying channel, the SNR at the output of despreader is $L$ times greater than the SNR at its input. In a multiple-access system, other users' signals interfere with the desired signal at the input to the receiver. In this situation, despreading performs the additional function of decorrelating multiple-access interference (MAI). The interfering signals have spreading codes that are orthogonal to the desired user's spreading code, and thus they are suppressed during despreading. In practice, however, the codes are not ideally orthogonal, leading to residual MAI. The level of residual MAI is determined both by the cross-correlation properties of the sequences and by the input power levels of the interferers and the desired user. An interferer whose received power is much higher than the desired user's power may create substantial residual in- 
terference even if the cross-correlation values are very low (but nonzero). This near-far situation can occur if the interferer is much closer to the receiver than the desired transmitter and the processing gain is not sufficient to offset the signal-to-interference ratio. To alleviate the near-far problem, direct-sequence spread-spectrum systems use power control. The various transmitters are told at which levels to transmit so that their signals arrive at the receiver with approximately equal powers. In addition to power control, multiuser receivers can be employed. These receivers attempt to detect not only the desired user's signal, but the signals of all active users. To do so, multiuser receivers use the knowledge of all the spreading codes. While they can attain excellent performance, their implementation complexity is extremely high. Multiuser receivers for underwater acoustic communications with very low processing gains have been investigated in [13]. Presently, we focus on two single-user receivers, the conventional despreader with symbol-rate phaselocked loop (PLL) plus gain control, and the chip-rate adaptive receiver with despreading performed at the equalizer output.

\section{A. DSSS Receiver With PLL and Automatic Gain Control}

To apply the conventional matched filter receiver to a multipath channel with varying phase several conditions must be met. One, to ensure that the ISI is not destructive, the symbol duration should be chosen to be much greater than the multipath spread of the channel, $T=L T_{c} \gg T_{m}$. If this condition is not met, there will be residual ISI at the output of the despreader and reduced receiver performance. Also, the despreader has no capability to track the phase variation typically found in the underwater channel. Thus, to make the conventional receiver applicable to the problem at hand, a PLL must be added. In addition, the power of the received signal varies with time and this must be tracked as well.

There are a number of methods for implementing a PLL [14]. The method proposed in this paper is a symbol-rate, decision-directed PLL suitable for use with an arbitrary M-ary linear modulation. Such a modulation provides the bandwidth efficiency needed in many underwater acoustic channels, while use of decision-direction in the PLL effectively uses available information from the equalizer and avoids squaring techniques that enhance noise and multi-user interference.

The performance of the PLL is governed by the choice of phase-tracking constants, or the loop filter parameters, which determine the loop bandwidth and the conditions for the loop stability [14]. The optimal values of these constants depend on the signal amplitude. For this reason, it is desirable that the input signal be of constant amplitude. Automatic gain control (AGC) is thus included immediately prior to the PLL.

The AGC is implemented by estimating the time-varying signal power and normalizing the signal accordingly. Let $w(t)$ be the output of the despreader which is provided to the AGC. The signal power $P=E\left\{\left|w^{2}(t)\right|\right\}$ can be estimated by computing the weighted time-average

$$
P(n)=(1-\alpha) \sum_{i=0}^{n} \alpha^{n-i}\left|w^{2}(i)\right|
$$

where $\alpha \leq 1$ is an exponential weighting factor. Taking the expected value of both sides of (1) and letting $n \rightarrow \infty$, we can verify that $P(n)$ is an asymptotically unbiased estimate of the power $P$ which is assumed to be wide-sense stationary. The expression for the power estimate (1) can be written in the form of a recursion

$$
P(n)=\alpha P(n-1)+(1-\alpha)\left|w^{2}(n)\right| .
$$

The power estimate is used to normalize the signal $w(n)$

$$
v(n)=\frac{w(n)}{\sqrt{P(n)}} .
$$

Neglecting ISI, and assuming perfect normalization, the output of the AGC can be modeled as

$$
v(n)=d(n) e^{j \theta(n)}+z(n)
$$

where

$d(n)$ data symbols from an independent and identically distributed unit-variance sequence;

$\theta(n)$ unknown phase;

$z(n)$ additive noise.

The decision-directed PLL produces an estimate of the data symbol as

$$
\hat{d}(n)=v(n) e^{-j \hat{\theta}(n)}
$$

where $\hat{\theta}(n)$ is the phase estimate. The error in data-symbol estimation is given by

$$
e(n)=d(n)-\hat{d}(n) .
$$

The data symbols $d(n)$, needed to compute the error, are known during the training period. After the training period, decisions $\widetilde{d}(n)$ are used in place of the true values $d(n)$. The decisions are made on the estimates $\hat{d}(n)$. In what follows correct decisions are assumed.

The task of the PLL is to form an estimate of the phase which will minimize the error in the mean square sense. This task will be accomplished when

$$
\frac{\partial E\left\{\left|e^{2}(n)\right|\right\}}{\partial \hat{\theta}}=-2 E\left\{\operatorname{Im}\left\{\hat{d}(n) e^{*}(n)\right\}\right\}=0 .
$$

The instantaneous value of this gradient defines the phase error

$$
\psi(n)=\operatorname{Im}\left\{\hat{d}(n) e^{*}(n)\right\}=\operatorname{Im}\left\{\hat{d}(n) d^{*}(n)\right\} .
$$

The data symbols needed to compute the phase error after the training period are obtained by making the decision on the estimates $\hat{d}(n)$. In the absence of decision errors

$$
\psi(n)=\operatorname{Im}\left\{v(n) e^{-j \theta(n)} d^{*}(n)\right\} .
$$

In the absence of noise, and with perfect gain control, the above expression can be written as

$$
\begin{aligned}
\psi(n) & =\sin [\theta(n)-\hat{\theta}(n)] \\
& =\sin \Delta \theta(n) \approx \Delta \theta(n) \text { for }|\Delta \theta(n)| \ll 1 .
\end{aligned}
$$




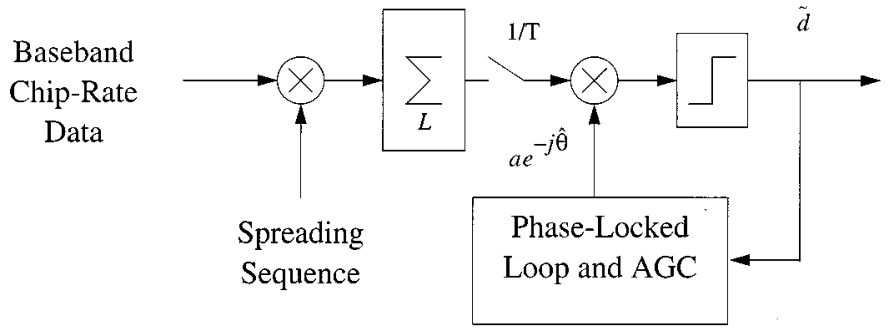

Fig. 1. Block diagram of the direct-sequence spread-spectrum receiver showing the standard despreading and decision process augmented by a decision-directed phase-locked loop with automatic gain control.

The phase error $\psi(n)$, computed with (8), is used with a secondorder filter to obtain the phase estimate

$$
\hat{\theta}(n+1)=\hat{\theta}(n)+K_{1} \psi(n)+K_{2} \sum_{i=0}^{n} \psi(i)
$$

where $K_{1}$ and $K_{2}$ are tracking constants chosen to provide the desired loop gain and bandwidth.

The complete PLL consists of (5), (6), (8) and (11). The PLL operates at the symbol rate, i.e., a new estimate of the phase is obtained only every $L$ chips. The phase-tracking performance is thus affected by the symbol duration and tracking is faster with shorter $L$, which may conflict with the requirement for $L$ to be longer than the multipath duration. The results of using the symbol-rate receiver (Fig. 1) on in-water data are discussed next. A modified receiver structure which removes this constraint is examined in the Section IV.

\section{B. DSSS Receiver With Chip-Rate Equalization}

The receiver described above tolerates phase and amplitude changes but is less tolerant of multipath. As already noted, the spreading code must be longer than the multipath delay in order to avoid intersymbol interference after despreading. However, to achieve the spreading gain $L$, the channel must be stationary over the code length. In a nonstationary environment, a longer code leads to larger phase and multipath variation over a code period, thus reducing the achievable spreading gain.

In order to improve the performance of DSSS in a nonstationary environment, an adaptive receiver structure in the form of a chip-rate decision feedback equalizer [9] is proposed (Fig. 2). The adaptive chip-rate equalizer reduces multipath and performs Doppler correction [10], prior to despreading. While the receiver introduces additional complexity by operating at the chip rate rather than the bit rate, the channel tracking capability is increased and otherwise unresolvable interchip interference is removed. This approach allows direct-sequence signaling to be used even when the delay spread exceeds the symbol length $L$.

An equally important feature of the adaptive chip-rate receiver is its ability to reduce interference from other users [2]. The filter taps are adapted using a minimum mean-square error criterion, which in the presence of interfering users attempts to suppress other users in addition to minimizing the effects of ISI. When multiple hydrophones are available the receiver provides

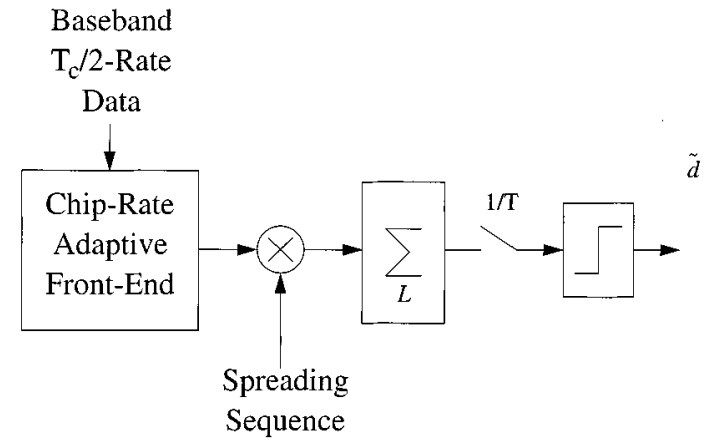

Fig. 2. Spread-spectrum receiver with chip-rate adaptive equalizer placed before the despreader.

MMSE combining and tends to focus the array on the user of interest through adaptation of the feedforward filters.

It was pointed out in Section III. A that direct-sequence systems are often operated at a very low SNR per chip, and that the spreading gain $L$ is used to extract the transmitted data. This means that reliable chip-rate decisions are not normally available for feedback in the equalizer. In a practical receiver, tentative chip decisions are needed to update the equalizer filters without delay. Some of the options for obtaining chip decisions are to make are: 1) hard decisions on the chip estimates; 2) hard decisions on the partially despread symbol; or 3) soft decisions on the partially-despread symbol [6]. A partially-despread data symbol is the running sum at the output of the matched filter receiver where reliability increases with summation length.

An alternative approach is to allow a one-symbol delay in updating the equalizer, though at a subsequent loss in tracking rate. In this method, the received signal is processed using equalizer coefficients from the previous symbol, then despread to estimate the actual transmitted chips, which are then fed back to update the filters. In what follows, perfect chip feedback is used to provide an upper bound to the performance of any practical feedback technique.

The decision feedback equalizer operates on the complex baseband signal $x(n)$ which is fractionally sampled at twice the chip rate. This signal is used with previous chip decisions $\tilde{c}$ to form the estimated chip value

$$
\hat{c}(n)=\Phi^{*}(n) W(n) e^{-j \hat{\theta}(n)}
$$

where $\Phi(n)=\left[x(2 n), x(2 n-1), \ldots, x\left(2 n-N_{f}\right), \tilde{c}(n-\right.$ $\left.1), \ldots, \tilde{c}\left(n-N_{b}-1\right)\right]$ and $N_{f}, N_{b}$ are the number of feedforward and feedback filter elements respectively. The filter vector $W(n)$ for the $n$th chip is a composite of $N_{f}$ feedforward and $N_{b}$ feedback weights and it is updated using the LMS or RLS algorithm [12]. The algorithm is driven by the chip error $e(n)=$ $c(n)-\hat{c}(n)$. The error is computed using either the true value of the chip $c(n)$ or the hard decision $\tilde{c}(n)$.

A chip-rate PLL is required to correct for Doppler shift which is inefficient for the equalizer alone to remove. The phase error for the current chip is computed using

$$
\psi(n)=\operatorname{Im}\left\{c^{*}(n) \hat{c}(n)\right\}
$$


TABLE I

Data Rate AND Symbol Time Summary FOR THE EXPERIMENTS

\begin{tabular}{c||c|c|c}
\hline Spread Factor & Data Rate & Symbol Time & FSK Bin Pairs \\
\hline 255 & $19 \mathrm{bps}$ & $53.1 \mathrm{~ms}$ & 70 \\
127 & $38 \mathrm{bps}$ & $26.5 \mathrm{~ms}$ & 35 \\
63 & $76 \mathrm{bps}$ & $13.1 \mathrm{~ms}$ & 17 \\
31 & $155 \mathrm{bps}$ & $6.5 \mathrm{~ms}$ & 8 \\
15 & $320 \mathrm{bps}$ & $3.1 \mathrm{~ms}$ & 4 \\
\hline
\end{tabular}

and the phase estimate $\hat{\theta}(n+1)$ for the next chip is updated using (11)

$$
\hat{\theta}(n+1)=\hat{\theta}(n)+K_{1} \psi(n)+K_{2} \sum_{i=0}^{n} \psi(i) .
$$

At the output of the equalizer, $L$ nonquantized chip estimates are despread to form the estimate of the data symbol

$$
\hat{d}(i)=\frac{1}{L} \sum_{m=(i-1) L}^{i L-1} \hat{c}(m)^{*} c(m) .
$$

The symbol decision is finally made by hard limiting (in the case of binary PSK) the above estimate

$$
\tilde{d}(i)=\operatorname{sgn}\{\hat{d}(i)\} .
$$

\section{EXPERIMENTAL RESULTS}

A series of tests were conducted at short range in shallow water to determine the performance of the various signals and receivers. A set of five FHSS and five DSSS signals were transmitted at rates varying from 19 bps to 320 bps using a center frequency of $13.5 \mathrm{kHz}$ and a $4800-\mathrm{Hz}$ bandwidth. The data rates and symbol durations are summarized in Table I. The signal power was approximately $183 \mathrm{~dB}$ re micro-Pascal. The FHSS and DSSS signals were sent in an alternating fashion from the slowest to the fastest with small delays between the packets. The purpose of transmitting the signals at five different data rates was to measure the performance with respect to spreading rate for both modulation types.

The frequency-hopped signals use binary frequency-shift keying and step sequentially through all frequencies while the direct-sequence signals use binary phase-shift keying and Gold codes [8] for spreading. The number of data bits per packet was fixed at 416. For the DSSS signals 1024 chips of training were included at the start of each packet. The entire set of signals spanned approximately $90 \mathrm{~s}$ and the tests lasted 30 to $45 \mathrm{~min}$. Coarse timing acquisition was achieved by matched-filtering to a 0.2 -s frequency-modulated signal transmitted prior to each DSSS or FHFSK packet. The FM sweep was also used to estimate the channel impulse response.

The direct-sequence signals were down converted from the $13.5-\mathrm{kHz}$ carrier to baseband and sampled at $9600 \mathrm{~Hz}$ which provides two samples per chip. The data were processed using both the symbol-rate matched-filter receiver and the chip-rate adaptive receiver described in the Section III.

Example impulse responses and spectra of the two time-varying channels are shown in Fig. 3. The response is highly reverberant in both cases, but with different decay constants. In both cases, the multipath produces a frequency-selective spectrum. These channels are relevant for shallow-water sampling networks or coastal AUV operations. Test 1 was performed in $20 \mathrm{~m}$ deep water over a $400 \mathrm{~m}$ path in an enclosed harbor, while Test 2 was performed over a $500 \mathrm{~m}$ path with depth ranging from $2 \mathrm{~m}$ to $6 \mathrm{~m}$. The data were recorded using a single broadband hydrophone and digitized for later laboratory analysis. The transmitter and receiver were not rigidly mounted, but were suspended from a pier and moved $10-30 \mathrm{~cm}$ with the prevailing currents.

\section{A. FHSS Results}

The FHSS signals were demodulated using a matched-filter bank after being synchronized to the FM signal. Hard decisions were made on the filter bank output and the number of bit errors was measured by comparison with the known data. Sufficient errors were observed with the FHSS signals so as to allow meaningful BER measurements down to $10^{-4}$.

The bit error-rate results obtained with the FSK signals are shown in Fig. 4. It is apparent that increasing the tone length improves the bit-error rate consistently for the very shallow water test (Test 2), while in the harbor (Test 1) the added energy provides less improvement at each reduction of data rate, and offers no improvement at the lowest rate. The reason for the performance difference is due to the nature of the acoustic channel. The harbor has the longest reverberation and the potential for the highest variability due to currents in the enclosed area. The very shallow water channel has more spectral nulls, but higher signal level than in the harbor.

Increasing the amount of energy per bit decreases the bit-error rate in very shallow water, while in the more reverberant harbor channel no additional gain is available when the time per symbol exceeds $26 \mathrm{~ms}$ ( 127 chips per symbol divided by 4800 chips per second). In channels such as this one, increasing the energy per bit is more appropriately done by fixing the tone bandwidth and increasing the coding gain to improve reliability, or by using fast-hopping where multiple tones are transmitted to convey one data symbol.

\section{B. Symbol-Rate DSSS Receiver Results}

Fig. 5 shows an example of the performance of the simple matched-filter DSSS receiver. Results of processing one packet each of spreading rates 31,63 , and 127 are shown. The $L=15$ code is too short with respect to the multipath delay and, hence, ISI cannot be neglected. The $L=255$ code is long with respect to the channel variation so that the AGC and phase tracking rate of $19 \mathrm{~Hz}$ are too low to keep up with changes in the channel. In both of those cases the receiver fails to converge.

While the receiver operates successfully using three of the five spreading rates from the very shallow water test, receiver performance is modest and there are always several bit errors per packet, resulting in an error rate of approximately $10^{-3}$ to 

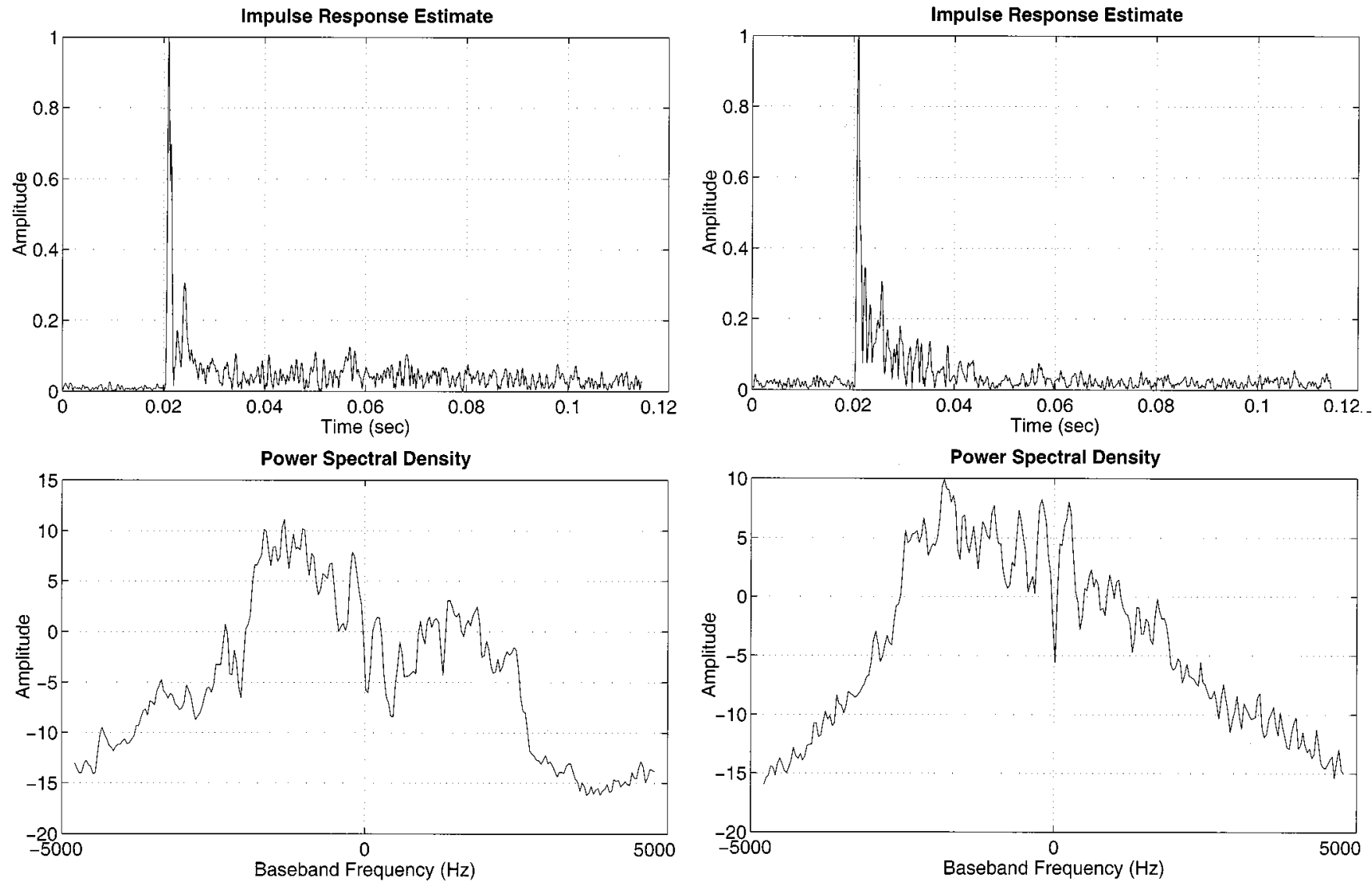

(a)

(b)

Fig. 3. Impulse response estimates and spectra from two shallow-water test sites. The impulse response estimates are obtained through matched filtering a 0.2 sec long $5 \mathrm{kHz}$ bandwidth LFM sweep centered at $13.5 \mathrm{kHz}$. The spectra are estimated by using a $4800 \mathrm{~Hz}$ BW PN sequence sampled at $9600 \mathrm{~Hz}$. (a) A $400 \mathrm{~m}$ link in $20 \mathrm{~m}$ deep water in an enclosed harbor. Note the very long delay response and frequency-selectivity.

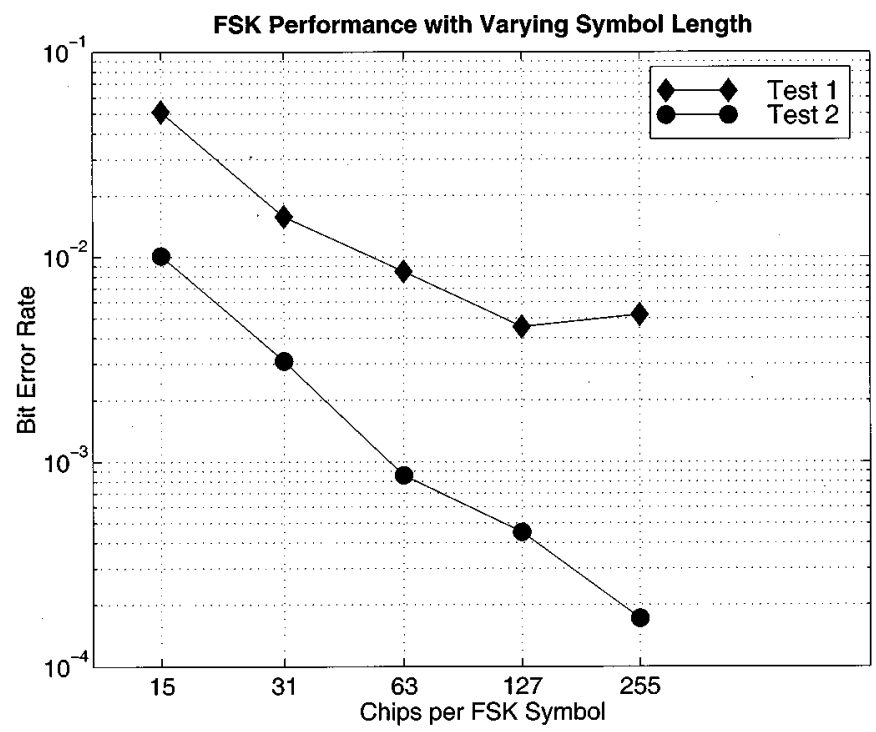

Fig. 4. Bit-error rate as a function of symbol duration spanning 15 to 255 chip intervals at $4800 \mathrm{~Hz}$ bandwidth for the acoustic channels shown in Fig. 3. Test 1 corresponds to the channel shown on the left in Fig. 3 which is the harbor test case, Test 2 is the very shallow-water test. The data rates shown are 320,155, 76,38 and 19 bits per second.

$10^{-2}$, which is slightly worse than the performance of FHSS at the same rate in the same channel.
The poor performance results from the fact that this receiver neglects multipath, and thus, residual ISI is present at the output of the despreader. The severity of the ISI depends on the code length: a shorter code results in a shorter bit interval which is more susceptible to the residual ISI. At the same time, the channel variation is tracked at the symbol rate and short code lengths allow faster amplitude and phase tracking. These two effects create conflicting requirements for the spreading rate. The resulting bit rate may not match communication system design needs in terms of throughput or the number of desired users.

\section{Chip-Rate DSSS Receiver Results}

The results obtained using the chip-rate adaptive DSSS receiver are shown in Fig. 6 where the output SNR after equalization and despreading is plotted versus the spreading rate. The measured error rate was near zero for both tests and, thus, it is more instructive to plot the output SNR than the bit-error rate. The adaptive filters are updated using perfect feedback so that the results shown here represent best-case processing with the chip-rate DFE. The achievable processing gain is now a function of residual phase distortion and irreducible ISI at the equalizer output.

For both test cases, the 255 chips per symbol rate offers little increase in output SNR over the 127 chips per symbol rate. This fact indicates that there is no performance improvement 

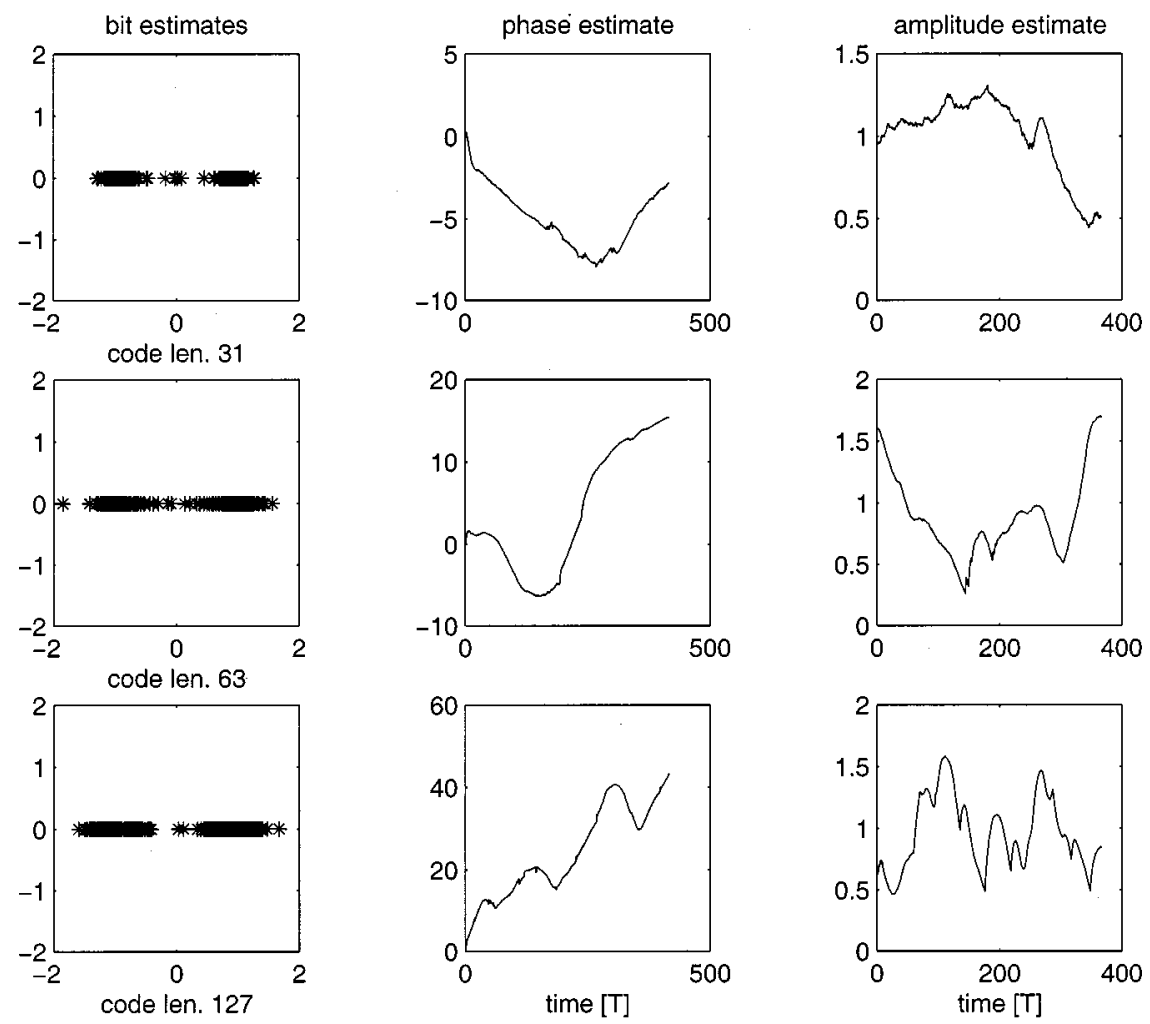

Fig. 5. Results from the symbol-rate receiver with PLL and AGC using code lengths 31, 63 and 127. In the first column are the scatter plots of bit estimates on which final decisions are made. In each instance there are a few (2-4) bit errors per packet; however, the receiver operation is not affected by error propagation. Three bits suffice to train the decision-directed PLL. The phase estimates are shown in the second column. The third column shows the signal amplitude estimated by the AGC. Both the phase and the amplitude estimates illustrate the rapid time-variation of the shallow-water channel.

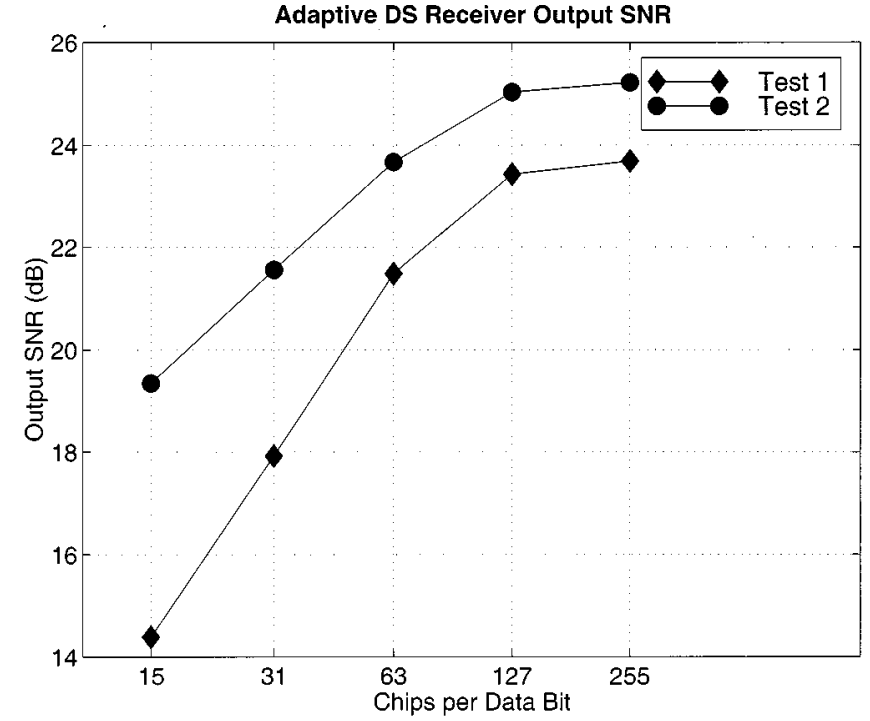

Fig. 6. SNR measured at the output of the despreader using the adaptive equalizer. Test 2 is the very shallow water case which offers better performance than the highly reverberant harbor. As with the FSK signals, the resulting data rates are $320,155,76,38$ and 19 bits per second for these spreading rates.

to be gained by reducing the symbol rate below 38 bps in this particular set of acoustic channels. In order to increase reliability at very low data rates, further redundancy is best introduced through error-correction coding rather than through longer spreading sequences.
Comparing the performance of the two DSSS receivers shows the superiority of the receiver that uses adaptive chip-rate processing. While certain benign channels may allow use of the matched-filter receiver, for flexibility in selecting the signaling rate, a chip-rate receiver is highly desirable. Alternative versions of the fully adaptive receiver are possible, and these are currently under investigation.

\section{CONCLUSION}

The performance of frequency-hopping and direct-sequence CDMA systems depends upon the nature of the acoustic channel. Experimental results obtained so far in shallow water point to the time-variability as the primary limitation to the performance of the conventional receiver. DSSS is shown to provide excellent performance when coupled with a chip-rate adaptive equalizer. Channel variability which is not removed by the adaptive equalizer ultimately determines the performance of the receiver at high spreading rates.

The symbol-rate receiver with PLL and AGC is sometimes adequate, but the range of conditions where it provides satisfactory performance may not be sufficient for multivehicle ocean sampling networks in shallow water. When the channel changes faster than the symbol rate, degradation in performance is observed and a chip-rate adaptive receiver is required.

The raw bit error rate of FHSS is higher than that of DSSS for the same SNR, though FHSS is very simple and thus attractive for certain applications. In addition, FHSS offers good intrinsic 
near-far resistance, in particular when used with convolutional coding and soft-decision Viterbi decoding.

This work has presented several candidate signaling methods and receivers suitable for undersea spread-spectrum acoustic communication. The single-user cases presented here will serve as a basis for future work that focuses on multiuser performance for undersea networks.

\section{REFERENCES}

[1] R. L. Peterson, R. E. Ziemer, and D. E. Borth, Introduction to SpreadSpectrum Systems. Englewood Cliffs, NJ: Prentice-Hall, 1995.

[2] S. Glisic and B. Vucetic, Spread-Spectrum CDMA Systems for Wireless Communication. Norwell, MA: Artech House, 1997.

[3] J. H. Fischer and R. Kendrick et al., "A high data rate, underwater acoustic data-communication transceiver," in Proc. Oceans '92, Newport, RI.

[4] G. Loubet, V. Capellano, and R. Filipiak, "Underwater spread-spectrum communications," in Proc. Oceans '97, Halifax, Canada.

[5] C. Boulanger, G. Loubet, and J. Lequepeys, "Spreading sequences for underwater multiple access communications," in Proc. Oceans '98, Nice , France.

[6] L. A. Rusch and H. V. Poor, "Narrowband interference suppression in CDMA spread spectrum communications," IEEE Trans. Commun., vol. 42, pp. 1969-1979, Apr. 1994.

[7] P. Fan and M. Darnell, Sequence Design for Communications Applications. Hertfordshire, U.K.: Research Studies Press, 1996.

[8] D. V. Sarwate and M. B. Pursley, "Crosscorrelation properties of pseudorandom and related sequences," Proc. IEEE, vol. 68, pp. 593-619, May 1980.

[9] M. Stojanovic, J. Catipovic, and J. Proakis, "Phase coherent digital communications for underwater acoustic channels," IEEE J. Oceanic Eng., vol. OE-16, pp. 100-111, Jan. 1994.

[10] M. Johnson, L. Freitag, and M. Stojanovic, "Improved Doppler tracking and correction for underwater acoustic communication," in Proc. ICASSP'97, vol. 1, Munich, Germany, Apr. 1997, pp. 575-578.

[11] J. Proakis, Digital Communications, 3rd ed. New York: McGraw Hill, 1995.

[12] L. Freitag, M. Johnson, and M. Stojanovic, "Efficient equalizer update algorithms for acoustic communication channels of varying complexity," in Proc. Oceans '97, Oct. 1997, pp. 580-585.

[13] M. Stojanovic and Z. Zvonar, "Multichannel processing of broadband multiuser communication signals in shallow water acoustic channels," IEEE J. Oceanic Eng., vol. 21, pp. 156-166, Apr. 1996.

[14] W. Lindsey and C. Chie, "A survey of digital phase-locked loops," IEEE Trans. Commun., pp. 410-431, Aug. 1989.

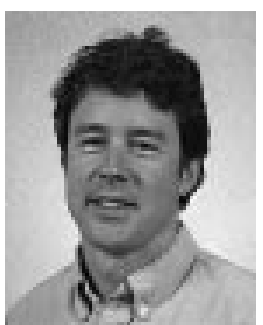

Lee E. Freitag received the B.S. and M.S. degrees in electrical engineering from the University of Alaska, Fairbanks.

He has worked for the past 12 years in underwater acoustic communication, navigation and tomography at the Woods Hole Oceanographic Institution, Woods Hole, MA. His research interests include single and multiuser acoustic communication systems suitable for vehicles and undersea networks.

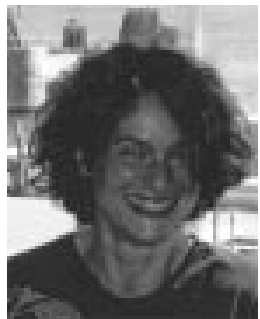

Milica Stojanovic received the Dipl. Ing. degree in electrical engineering from the University of Belgrade, Belgrade, Yugoslavia, in 1988, and the M.S. and Ph.D. degrees in electrical engineering from Northeastern University, Boston, MA, in 1991 and 1993.

Currently, she is a Principal Scientist with the Massachusetts Institute of Technology, Cambridge, MA, and also serves as a Guest Investigator at the Woods Hole Oceanographic Institution, Woods Hole, MA. Her research interests include digita communications theory and statistical signal processing, with related problems in mobile radio, satellite, and underwater acoustic communications.

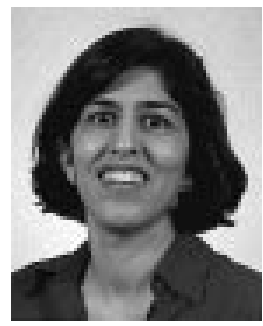

Sandipa Singh received the B.S. degree in electrical and computer engineering from George Mason University, Fairfax, VA, in 1989, and the M.S.degree in electrical engineering from University of Virginia, Charlottesville, in 1991.

She is an Electrical Engineer at Woods Hole Oceanographic Institution, Woods Hole, MA. She programs and maintains communications software for underwater acoustic modems.

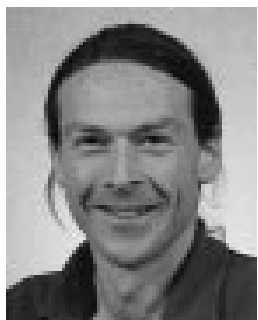

Mark Johnson was born in U.K. He received the Ph.D. degree from the University of Auckland, Auckland, New Zealand, in 1992

Since 1993, he has been a Research Engineer at Woods Hole Oceanographic Institution, Woods Hole, MA, where he is involved in developing underwater acoustic communication systems and acoustic tags for marine mammals. His research interests are in miniature signal processing instruments, bioacoustics, and algorithm design. 